\title{
Continuous laryngoscopy quantitates laryngeal behaviour in exercise and recovery
}

\author{
J. Tod Olin ${ }^{1}$, Matthew S. Clary², Elizabeth M. Fan ${ }^{1}$, Kristina L. Johnston ${ }^{3}$, \\ Claire M. State ${ }^{1}$, Matthew Strand ${ }^{4}$ and Kent L. Christopher ${ }^{5}$
}

Affiliations: ${ }^{1}$ Dept of Pediatrics, National Jewish Health, Denver, CO, USA. ${ }^{2}$ Dept of Otolaryngology, University of Colorado School of Medicine, Aurora, CO, USA. ${ }^{3}$ Dept of Rehabilitation Medicine, National Jewish Health, Denver, CO, USA. "Division of Biostatistics and Bioinformatics, National Jewish Health, Denver, CO, USA.

${ }^{5}$ Dept of Medicine, University of Colorado School of Medicine, Aurora, CO, USA.

Correspondence: J. Tod Olin, Dept of Pediatrics, National Jewish Health, 1400 Jackson Street, Box J-317, 80206, Denver, CO, USA. E-mail: olintanjhealth.org

ABSTRACT Exercise-induced laryngeal obstruction (E-ILO) causes exertional dyspnoea. There is no standardised methodology which characterises laryngeal obstruction in relation to exercise or links laryngeal obstruction and dyspnoea severity. Continuous laryngoscopy during exercise (CLE) may improve diagnostic sensitivity by enabling laryngeal visualisation at peak work capacity in patients with rapidly resolving obstruction. The time course of laryngeal obstruction across exercise and recovery has not been quantitated until this report.

Adolescents and young adults referred for CLE were laryngoscopically monitored across rest, maximal cycle ergometry exercise, and recovery. Three reviewers, blinded to time sequencing, rated inspiratory glottic and supraglottic obstruction during 10 windows of 15 -s corresponding to rest, $25 \%, 50 \%, 75 \%, 90 \%$ and $100 \%$ of individual symptom-limited peak work capacity (expressed in Watts), and four consecutive recovery windows.

85 patients were screened and 71 included. Over $96 \%$ of time windows were interpretable. Laryngeal obstruction severity reached observed maximal levels at peak work capacity, and rapidly resolved. A spectrum of observed maximal obstruction was measured.

CLE provides interpretable data demonstrating laryngeal obstruction in patients with suspected E-ILO that is more severe at peak work capacity than during rest, submaximal exercise, or recovery. Observed maximal obstruction was infrequently severe and rapidly resolved.

@ERSpublications

Maximal laryngeal obstruction occurs at peak work capacity in suspected exercise-induced laryngeal obstruction http://ow.ly/pHER300eiU9

This article has supplementary material available from erj.ersjournals.com

Received: Jan 212016 | Accepted after revision: May 01 2016 | First published online: July 132016

Conflict of interest: Disclosures can be found alongside the online version of this article at erj.ersjournals.com

Copyright OERS 2016 


\section{Introduction}

Exercise-induced laryngeal obstruction (E-ILO) is the preferred consensus-opinion term describing the condition in which glottic and/or supraglottic structures inappropriately obstruct the larynx during exercise, "causing breathing problems." [1] First described in the 1980s, E-ILO is important because it may affect a large number of adolescents and young adults and can mimic exercise-induced asthma (leading to inappropriate treatment) [2-8]. The diagnosis of E-ILO is confirmed via visualisation of laryngeal obstruction in relation to exercise. Diagnosis also depends on the temporal linking of any observed laryngeal obstruction with exertional dyspnoea [1].

Though laryngoscopy is considered the gold standard for diagnosis of inducible laryngeal obstruction (ILO), historically there has been variability in diagnostic methods used for evaluating laryngeal obstruction with exercise [1]. This is an important problem because studies designed to identify E-ILO phenotypes, mechanisms and treatments depend on diagnostic techniques that can quantitatively or semi-quantitatively establish the presence or absence of abnormal laryngeal obstruction. Diagnostic methods used in practice and described in the literature include documentation of a characteristic history, noninvasive techniques, resting laryngoscopy, post-exercise laryngoscopy, pre- and post-exercise laryngoscopy, and continuous laryngoscopy during exercise (CLE) [5, 9-12].

The CLE procedure has been advocated as the diagnostic technique of choice by authors at some centres [13]. The procedure features a flexible laryngoscope that is mounted in place throughout an exercise challenge [5]. It has been performed in a variety of exercise modes, including treadmill and cycle ergometry $[5,14,15]$. The procedure presents logistic challenges in terms of creating a representative exercise challenge and tolerable patient experience while simultaneously acquiring high-quality continuous laryngoscopic data. However, the hypothesised benefit of CLE in documenting laryngeal obstruction is a decrease in false negative examinations as it allows for laryngeal visualisation during strenuous exercise in patients whose obstruction resolves over brief time periods that preclude documentation through post-exercise visualisation. Additionally, the procedure is hypothesised to minimise false positive examinations that could be caused by the introduction of a laryngoscope during periods of appropriate dyspnoea immediately after strenuous exercise [14].

While the timing of the onset and resolution of laryngeal obstruction are thought to be important, there is a current gap in the literature regarding the dependence of upper airway obstruction in affected patients on work rate and recovery time [1]. An understanding of these relationships will shed light on the mechanisms underlying the condition and improve understanding of the sensitivity of several diagnostic procedures used to confirm the clinical diagnosis of E-ILO.

The purpose of this study was to elucidate the relationship between upper airway obstruction at a glottic and/or supraglottic level and both work rate and recovery time. We asked the specific question: is laryngeal obstruction more likely to be observed while subjects exercised at symptom-limited individually-achieved peak work capacity (which for the purpose of this investigation is herein defined as peak work rate, expressed in Watts) than during time windows that can be observed with traditional laryngoscopic methods (including resting laryngoscopy, laryngoscopy after submaximal exercise and laryngoscopy $1 \mathrm{~min}$ after peak exercise)?

We hypothesised that observed upper airway obstruction would reach maximally observed levels at symptom-limited individually-achieved peak work capacity and would rapidly resolve during recovery (in less than $60 \mathrm{~s}$ ).

The main study objective was to characterise this relationship by describing inspiratory glottic and supraglottic obstruction across rest, incremental exercise to symptom-limited individually-achieved peak work capacity, and recovery in a retrospective group of consecutive adolescent and young adult patients who underwent CLE to evaluate exertional dyspnoea.

\section{Materials and Methods}

\section{Subjects}

The National Jewish Health Institutional Review Board approved this retrospective study. We included 85 sequential patients, aged 12 through 21, who underwent CLE for any indication between March, 2013 and February, 2014. We then excluded patients who were referred for CLE for indications other than clinical suspicion for E-ILO and patients with test datasets that could not be analysed due to technical failure of specific devices.

\section{Continuous laryngoscopy during exercise apparatus}

The details of our CLE apparatus have been described (see the online supplementary material for hardware) [16]. In summary, a helmet-mounted flexible fiberoptic laryngoscope was fixed near the nostril to minimise distance changes between the laryngoscope and larynx. 


\section{Exercise protocol and data acquisition}

After $3 \mathrm{~min}$ of rest and $3 \mathrm{~min}$ of unloaded cycling, patients performed ramp protocols (continuously increasing work without discrete stages) designed to reach peak work capacity within 8-12 min followed by recovery [17]. The tests were symptom-limited and terminated when patients could no longer continue to cycle despite vigorous verbal encouragement. Heart rate, ventilation and metabolic data (oxygen uptake and carbon dioxide output) were recorded at $200 \mathrm{~Hz}$.

\section{Video acquisition and sampling}

The laryngoscopy video, time-synchronised with metabolic data collection (via audio cues), was recorded across rest, exercise and recovery. 10 specific 15-s time windows were extracted at rest, 25\%, 50\%, 75\%, $90 \%$ and $100 \%$ of achieved peak work capacity as well as at periods centred at 15, 30, 45 and $60 \mathrm{~s}$ into recovery. We did not correct for image distortion [18].

\section{Video scoring and data compilation}

Three blinded reviewers (a physician's assistant in paediatric pulmonology, an otolaryngologist and a speech and language pathologist all familiar with the procedure) independently scored the 10 windows of each test for video quality, inspiratory glottic obstruction and inspiratory supraglottic obstruction. Expiratory findings were not scored. Reviewers scored obstruction across an entire window based on a four-point visual scoring scale published by RoKsund et al. [19] which characterises glottic and supraglottic behaviour as normal (0), mild (1), moderate (2) and severe (3). Reviewers scored all windows for individual subjects in a single session because differences in laryngoscope placement can affect measurements [18]. Within each subject, reviewers first scored the video acquired at $25 \%$ peak work capacity to establish a reference because, based on pilot data, this is a window in which laryngeal obstruction is rare [20]. Reviewers scored the remaining nine videos blinded to time sequencing via randomisation and muting of the audio track.

We compiled data as follows. We excluded data from windows in which two or more reviewers noted that visual obstruction or vocalisations prevented analysis. If two or more reviewers scored a video as interpretable, we averaged scores to quantitate obstruction as a numeric value. When qualitatively describing an individual's peak laryngeal obstruction on an ordinal scale in descriptive portions of the report, we rounded average scores to the nearest integer (rounding down in scenarios when the average of only two interpretable reviews was midway between two integer values in order to avoid overstating obstruction).

\section{Statistical analysis}

Descriptive statistics were computed by reviewer and time window, including means, standard errors and percentage of agreement (for pairs of reviewers) or percentage of agreement within one unit (for all three reviewers). Kendall's W statistic was also used to assess agreement between reviewers. Differences between windows were compared by fitting a longitudinal model for ordinal score, using Poisson regression, log link, employing generalised estimating equations with a first-order autoregressive working correlation structure for window; window, reviewer and window-by-reviewer interaction were included in the model as fixed-effect terms. As Poisson regression does not impose an upper bound to the outcome (and our outcome has an upper bound), other models were also fitted to verify results (see the online supplementary material).

\section{Results}

Patients

A total of 85 patients were included in the screening. Six (7\%) were excluded due to the technical inability to time synchronise the exercise and laryngoscopic data. Eight (9\%) were excluded as the reason for referral was not exertional dyspnoea (refractory asthma, refractory ILO and an atypical presentation for anaphylaxis). Demographic data are summarised in table 1 . This was a young population by definition with a female predominance. The majority of patients were Caucasian. The study population had a mean body mass index and mean peak oxygen consumption similar to the general population [21, 22]. There was notable variability in peak oxygen consumption that appeared to be due to an upward skew in its distribution. Nine subjects had peak oxygen consumption above $50 \mathrm{~mL} \cdot \mathrm{kg}^{-1} \cdot \mathrm{min}^{-1}$. Peak work capacity ranged from $96 \mathrm{~W}$ to $352 \mathrm{~W}$ across our population.

\section{Scoring methodology}

The majority of video windows were considered interpretable by at least two reviewers (table 2). During exercise and recovery, $93 \%$ or more of the videos in all specific windows were interpretable. At peak work capacity, reviewers were able to interpret glottic and supraglottic behaviour in $97 \%$ and $99 \%$ of the videos respectively. At rest, reviewers were able to interpret glottic behaviour in $90 \%$ of videos. Secretions and vocalisations were the artefacts that limited interpretability (and were more common at rest). 


$\begin{array}{lc}\text { TABLE } 1 \text { Patient demographics and clinical features } & \\ \text { Age years } & 15.0 \pm 2 \\ \text { Female sex } & 47(66) \\ \text { Race/ethnicity } & 64(90) \\ \quad \text { Caucasian } & 3(4) \\ \text { African-American } & 2(3) \\ \text { Hispanic } & 2(3) \\ \quad \text { Asian } & 21.7 \pm 3.6 \\ \text { Body mass index } \mathbf{~ k g} \cdot \mathbf{m}^{-\mathbf{2}} & 197 \pm 60 \\ \text { Peak work Watts } & 39.3 \pm 9.8 \\ \text { Peak } \mathbf{V O}_{\mathbf{2}} \mathbf{~} \mathbf{~ L L} \cdot \mathbf{k g}^{-\mathbf{1}} \cdot \mathbf{m i n}^{-\mathbf{1}} & \end{array}$

Data are presented as mean \pm SD or $\mathrm{n}(\%)$. Peak $\mathrm{VO}_{2}$ : peak oxygen consumption.

Overall agreement across reviewers was strong. $97 \%$ and $92 \%$ of individual windows were characterised by reviewer agreement (scored within severity 1 unit across all reviewers) for glottic and supraglottic obstruction, respectively. While general trends regarding the rating of glottic and supraglottic obstruction were consistent across reviewers, there was somewhat more variability at high work rates (figure 1). At $25-50 \%$ of peak work capacity, reviewer agreement was $98 \%$ for both glottic and supraglottic obstruction respectively. Agreement nadirs based on time window were $90 \%$ for glottic scoring (at peak work capacity) and $87 \%$ for supraglottic scoring (at $30 \mathrm{~s}$ into recovery). Kendall's W ranged from 0.40 and 0.86 for glottic assessments ( $\mathrm{p}<0.01$ for all except for the second time window: $\mathrm{p}=0.08$ ) and from 0.47 to 0.78 for supraglottic assessments across time windows $(\mathrm{p}<0.01$ for all), indicating moderate-to-strong agreement between reviewers ( 0 indicates no trend for agreement).

\section{Obstruction severity}

A spectrum of observed maximal obstruction severity was measured at both a glottic and supraglottic level (table 3). Inspiratory glottic obstruction was characterised as moderate in 26 (37\%) and severe in six (8\%) subjects during at least one window of the testing period. Inspiratory supraglottic obstruction was characterised as moderate or severe in $53(75 \%)$ subjects. $33(46 \%)$ subjects were characterised as having mild inspiratory glottic obstruction (at the individual's peak severity), with two of these subjects demonstrating resting findings only. The combination of inspiratory glottic and supraglottic obstruction was seen in the majority of subjects (table 4). No subjects demonstrated severe obstruction at one level without some degree of obstruction at the other level.

\section{Obstruction timing in the sample}

The time course of obstruction severity in the group as a whole was notable (figure $2 \mathrm{a}$ and $\mathrm{b}$ ). Neither glottic nor supraglottic obstruction was prominent at rest or work rates $<75 \%$ of peak work capacity. When the sample was narrowed to those with moderate or severe inspiratory glottic obstruction, the general trend was similar, although estimates were higher near peak work capacity (figure $2 \mathrm{c}$ and $\mathrm{d}$ ). The severity of obstruction at both a glottic and supraglottic level reached their observed maximal levels at peak work capacity. There were significant differences in obstruction severity at a glottic and supraglottic level when peak work capacity was compared with $90 \%$ peak work capacity ( $\mathrm{p}<0.0001$ for both) in the overall subject sample. When the sample was restricted to subjects with moderate or severe obstruction, this comparison yielded similar results $(\mathrm{p}<0.0001$ for both). More notable differences were measured between peak work capacity and all lower levels of submaximal exercise (in both the general sample of

\begin{tabular}{|c|c|c|c|c|c|c|c|c|c|c|}
\hline \multirow[t]{2}{*}{ Obstruction } & \multirow[t]{2}{*}{ Rest } & \multicolumn{5}{|c|}{ Peak work capacity \% } & \multicolumn{4}{|c|}{ Recovery time s } \\
\hline & & 25 & 50 & 75 & 90 & 100 & 15 & 30 & 45 & 60 \\
\hline Glottic & 90 & 97 & 96 & 100 & 99 & 97 & 94 & 94 & 96 & 97 \\
\hline Supraglottic & 96 & 99 & 97 & 100 & 99 & 99 & 94 & 93 & 96 & 99 \\
\hline
\end{tabular}

Data are presented as \% of windows scored as interpretable by at least two of three reviewers across rest, incremental exercise and recovery. 

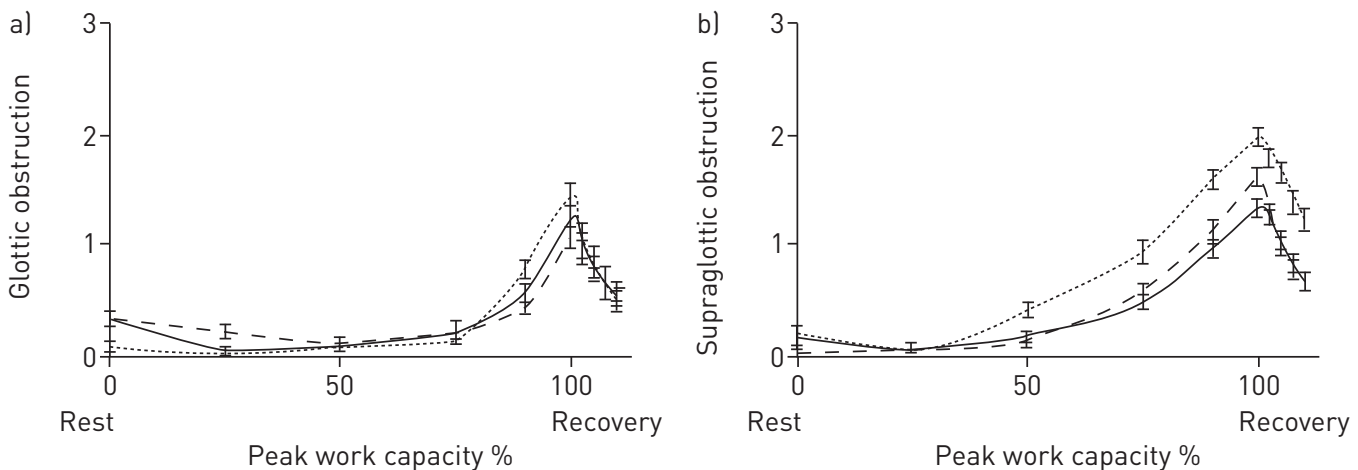

FIGURE 1 Raw inter-rater comparisons of a) glottic and b) supraglottic obstruction across rest, incremental ramp exercise to a symptom-limited peak and recovery in all subjects. Solid, dashed and dotted lines represent the average obstruction assessments across each of three reviewers, respectively, with standard errors noted. Data points represent the average obstruction ratings across all patients as per the scoring scale of RoKSUND et al. [19] with larger numbers representing increased obstruction. While differences are noted across reviewers at high work rates and during recovery, general trends were consistent across reviewers.

patients as well as in the sample with moderate-to-severe obstruction). Glottic obstruction rapidly resolved in both the general sample and in the sample of patients that demonstrated moderate to severe obstruction. Our pre-specified comparison between obstruction at peak capacity and 1 min into recovery reached statistical significance for both the glottis and supraglottis $(p<0.0001$ for both). Significant statistical differences were also noted in glottic obstruction between peak work capacity and the windows centred at 30 and $45 \mathrm{~s}$ into recovery as well ( $\mathrm{p}=0.0096$ and 0.0001 respectively). The resolution of supraglottic obstruction was also rapid, with statistically significant differences between peak work capacity and the windows centred at 15,30 and $45 \mathrm{~s}$ into recovery $(\mathrm{p}=0.031, \mathrm{p}<0.0001$ and $\mathrm{p}<0.0001$ respectively). Figure s1 (in online supplementary material) shows that averages for glottic and supraglottic outcomes based on raw data were similar to modelled estimates displayed in figure 2.

\section{Observation timing within individuals}

When the observation windows were evaluated within each individual, a similar trend emerged (table 5). The window of observed maximal obstruction severity was more likely to be the window of peak work capacity than windows correlating with rest, $90 \%$ peak work capacity, or any window in recovery.

\section{Discussion}

This is the first report that quantitatively defines the dependence of laryngeal obstruction on work rate and recovery time. The likelihood of observing laryngeal obstruction of any degree at either a glottic or supraglottic level is highly dependent on the conditions under which the observation is made. In this study, we present a clinical sample of adolescents and young adults that raised clinical concern for E-ILO. We demonstrate that, in the group as a whole, laryngeal obstruction at peak work capacity is more severe than obstruction during rest, submaximal exercise, and recovery. We demonstrated, within individual tests, the likelihood of observed maximal obstruction was highest at peak work capacity. We also demonstrated that, even in a highly biased population studied with CLE, the likelihood of observing severe glottic obstruction was low and that observed obstruction rapidly resolved.

\section{Significance of the findings}

This description of laryngeal obstruction across rest, exercise, and recovery may impact the manner in which clinicians and researchers document laryngeal obstruction during exercise. In current clinical practice, there

\begin{tabular}{lcccc}
\multicolumn{2}{l}{ TABLE 3 Distribution of maximal inspiratory laryngeal obstruction } & Severe \\
\hline & None or minimal & Mild & Moderate & $6(8)$ \\
\hline Glottic & $6(8)$ & $33(46)$ & $26(37)$ & $2(3)$ \\
Supraglottic & $3(4)$ & $15(21)$ & $51(72)$ & 2
\end{tabular}

Data are presented as $\mathrm{n}(\%)$ of patients in whom observed maximal obstruction at a glottic and supraglottic level was characterised as none/minimal, mild, moderate and severe respectively. 
TABLE 4 Coexistence of glottic and supraglottic obstruction

\begin{tabular}{lcccc} 
Inspiratory glottic obstruction & \multicolumn{3}{c}{ Supraglottic obstruction } \\
\cline { 2 - 5 } & None or minimal & Mild & Moderate & Severe \\
\hline None or minimal & 1 & 3 & 2 & 0 \\
Mild & 2 & 6 & 25 & 0 \\
Moderate & 0 & 5 & 20 & 1 \\
Severe & 0 & 1 & 4 & 1 \\
\hline
\end{tabular}

Data are presented a $\mathrm{n}$ patients in whom observed maximal supraglottic obstruction was characterised as none/ minimal, mild, moderate and severe respectively in patients stratified by the severity of glottic obstruction.

is noted heterogeneity in diagnostic techniques for this condition. In the literature, this condition is diagnosed via clinical history, resting laryngoscopy, post-exercise laryngoscopy, pre-and post-exercise laryngoscopy, and CLE. We believe that visualisation of laryngeal obstruction is required to diagnose E-ILO, especially in research samples. Furthermore, we believe that these are compelling data supporting CLE as the preferred diagnostic method for documenting laryngeal obstruction for two reasons. First, it seems clear that one is much more likely to observe obstruction at peak work capacity than during any other window of time. Second, observed maximal obstruction is not necessarily synonymous with total obstruction or a clinically significant degree of obstruction. Incomplete obstruction may be much easier to interpret in the context of multiple time periods of lower degrees of obstruction that CLE provides.

\section{Findings in the context of previous studies}

Continuous laryngoscopy during exercise is a relatively new procedure. Our findings are similar to a qualitative description (without quantitative support) of the rapid obstruction resolution [14]. Authors have advocated for the use of CLE for its effects in minimising theorised false positive findings in anxious patients post-exercise [5]. Although we did not present data quantitating the effect of laryngoscope
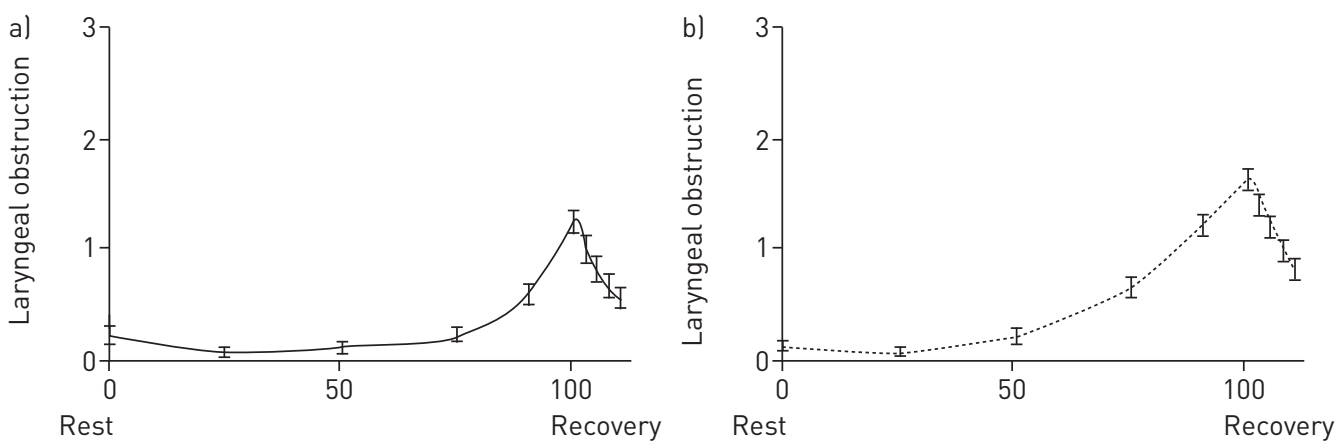

Peak work capacity \%

Peak work capacity \%
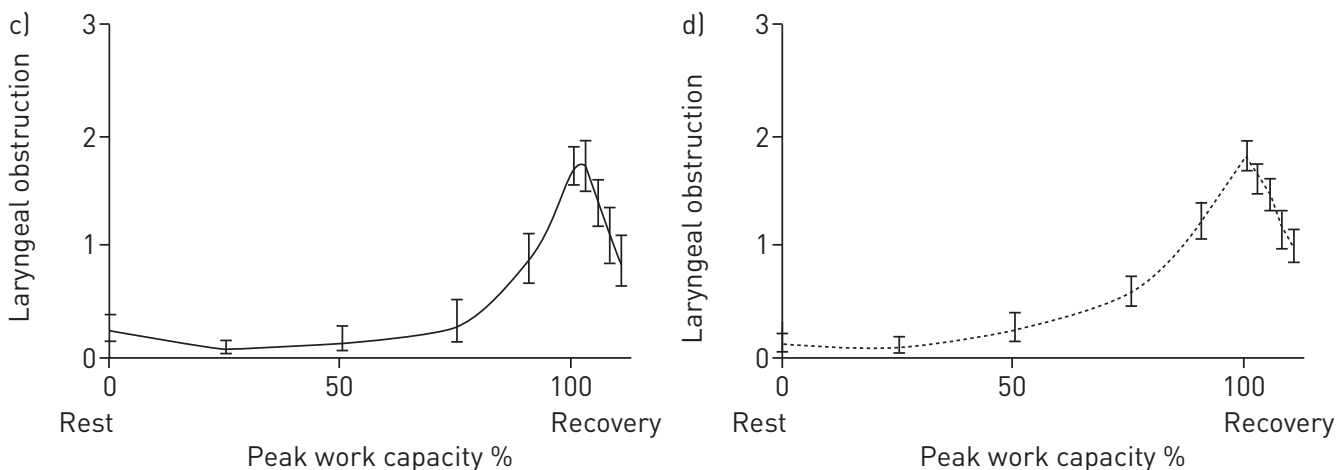

FIGURE 2 Inspiratory laryngeal obstruction across rest, incremental ramp exercise to a symptom-limited peak and recovery. Laryngeal obstruction in the entire group is quantitated at the level of the al glottis and b) supraglottis, respectively. Laryngeal obstruction in subjects with moderate or severe inspiratory glottic adduction in any test window is also quantitated at the level of the $\mathrm{c}$ ) glottis and d) supraglottis, respectively. Confidence intervals surround estimates and are based on the scoring scale of Roksund et al. [19] and the statistical model (see Statistical Analysis section in Materials and Methods and the online supplementary material for detail). 


\begin{tabular}{|c|c|c|c|c|c|c|c|c|c|c|}
\hline \multirow[t]{2}{*}{ Airway location } & \multirow[t]{2}{*}{ Rest } & \multicolumn{5}{|c|}{ Peak work capacity \% } & \multicolumn{4}{|c|}{ Recovery time s } \\
\hline & & 25 & 50 & 75 & 90 & 100 & 15 & 30 & 45 & 60 \\
\hline Glottic (n=65) & 2 & 0 & 0 & 2 & 7 & 44 & 18 & 10 & 9 & 7 \\
\hline Supraglottic $(n=68)$ & 1 & 0 & 1 & 5 & 20 & 50 & 25 & 14 & 11 & 7 \\
\hline
\end{tabular}

Data are presented as $\mathrm{n}$ continuous laryngoscopy during exercise tests in which the specific time windows were rated as maximally obstructed. In tests with more than a single window scored with observed maximal obstruction, each window is recorded. Patients with no observed obstruction by a specific structure are not included.

placement on glottic configuration, we anecdotally note that procedural anxiety is an important consideration especially in paediatric populations.

\section{Challenges of the testing environment}

The study of E-ILO is in its infancy and much work remains before there will be data-driven guidelines on which to base diagnostic and research strategies. Although the data quantified the relationships between laryngeal obstruction during exercise and both work rate and recovery time, it is possible that laryngeal obstruction is dependent on much more than exercise intensity.

Laryngeal obstruction during exercise may vary across patient populations. Here, we presented a clinical group of adolescent and young adult patients suspected of having E-ILO based on the clinical history which included the response to previous treatments. It is possible that other populations will demonstrate slightly different findings. The presence or absence of severe lower airways disease may be an important contributor [23].

Laryngeal obstruction during exercise may vary across exercise mode and protocol. It is difficult to administer a uniform exercise stimulus to a large population of patients and simultaneously maximise the likelihood of observing obstruction in a given individual. In the case of this study, we used an incremental ramp protocol to a symptom-limited peak, often used to quantitate cardiopulmonary function. In terms of this study, a benefit of this approach is that it allows for a precise quantification of work rate. However, it is possible that the likelihood of observed E-ILO would increase in testing environments that utilise treadmill ergometry or sprint exercise.

The likelihood of observing E-ILO may also vary across testing environments or other individual factors. It can be speculated that multiple phenotypes of patients with E-ILO might exist differing in potential factors such as laryngeal pliability, laryngeal reactivity, lower airways disease and behavioural responses to external motivation and situational stress.

\section{Challenges in data analysis}

Data analysis presents a second major challenge. As with any new technology which allows for increased data acquisition, there is now a need for improved data processing. At this time, there are no widely-available metrics which can precisely quantitate obstruction. Inter-rater reliability in subjective airway assessments has been previously demonstrated [24]. Some authors have advocated for the use of the EILOMEA software programme to measure specific, manually-identified laryngeal structures in isolated still frame images which has shown to correlate well with subjective assessments of individual respiratory cycles $[25,26]$. In the case of this study, we preferred a semiquantitative approach that accounted for thousands of frames of video, referenced within an individual to a time segment of minimal obstruction. With this approach, we noted significant differences in upper airway assessment across reviewers, but we prospectively selected an analytical model designed to take into account these differences. However, our approach, which was designed to minimise bias in the research setting, cannot be easily applied in the clinical arena (where a single subjective characterisation of laryngeal obstruction in term of the Roksund scale is most feasible at this time) [19].

Outside of specific quantitation of video findings, there is also a very important need to link observed findings with clinical significance. In our group of patients, the significance of the CLE findings and final diagnoses were based in part on recognised bias in the group (a generally fit population often specifically referred for their lack of clinical responsiveness to asthma therapy) and the sum of available clinical data acquired previously (which included at least one history and physical and resting spirometry in all cases, bronchodilator testing in $96 \%$ of cases and methacholine challenges in $10 \%$ of cases at our specialty referral institution). Our group biases are notable for asthma diagnosed by strict criteria (12\% improvement in 
forced expiratory volume in $1 \mathrm{~s}$ with bronchodilator administration or $20 \%$ decline with methacholine administration of $4 \mathrm{mg} \cdot \mathrm{min}^{-1}$ or less) in $15 \%$ of patients. We diagnosed all patients with moderate and severe obstruction at peak work capacity with EILO and diagnosed some patients with lower levels of visualised obstruction with EILO based on our clinical judgment. The presence or absence of strict asthma diagnosis did not affect the general contour of the obstruction versus work rate relationship (data not shown). While we feel that the likelihood of observing laryngeal obstruction during CLE is strongly dependent on the clinical history (and possibly ancillary tests), we feel that, if laryngeal obstruction is observed, the likelihood of observing maximal laryngeal obstruction at peak work capacity is very high.

However, there are few published studies linking precise quantitation of obstruction with symptoms or physiologic changes of interest [19]. In our sample, we demonstrate significant statistical changes in laryngeal obstruction across time, but we specifically avoided commenting on the degree of relative obstruction required to cause symptoms or impair performance, which may vary across individuals and exercise requirements. Our group of patients demonstrated a high frequency of (at least mild) obstruction at either a glottic or supraglottic level, a finding conceptually similar to data from previously-published referral groups, but higher than in groups representing general populations $[6,19]$. We feel that both bias in the reason for referral and observable mild findings of unclear significance in general populations likely contribute to our data, but we cannot quantitate the relative impact of each. Moreover, the significance of the relative contributions of supraglottic and glottic obstruction has yet to be precisely defined. Early literature initially described abnormal flow volume loops in patients with E-ILO [4]. More recently, the lack of correlation between CLE observations and pre-and post-exercise flow-volume loops has been described, as well as a lack of correlation between CLE observations and flow loops obtained during exercise in some fit individuals $[16,27,28]$. Intuitively, one might think that clinically significant laryngeal obstruction might impact ventilatory mechanics. We specifically avoided this topic, choosing to focus solely on observed obstruction. However, this is clearly a subject that requires further study with a goal of scientifically identifying the contribution of laryngeal obstruction to exertional breathing problems.

\section{Conclusion}

In our sample, glottic and supraglottic behaviour varied across rest, incremental exercise and recovery. Observed maximal laryngeal obstruction occurred at peak work capacity. The resolution of laryngeal obstruction during recovery was rapid (starting in less than $30 \mathrm{~s}$ ). This data may impact the diagnostic strategies used in E-ILO and present a compelling rationale to use CLE for the documentation of laryngeal obstruction in clinical and research samples. Moving forward, CLE may also quantitatively link laryngeal obstruction with dyspnoea.

\section{Acknowledgements}

The authors acknowledge all contributors in the Upper Airway Working Group, based at National Jewish Health (Denver, CO, USA) for their insights into this and future projects. The authors thank Erwin Gelfand, Leland Fan (Children's Hospital Colorado, Aurora, CO, USA), Michael Morris (San Antonio Military Medical Center, San Antonio, TX, USA), and Herman Staudenmayer (Denver, CO), for their thoughtful reviews of the manuscript and process. The authors also thank George Zeman, Valerie Keever, Meg Baldwin, Brenda Cox, Mark Fleming, Dave Gurka, and Lisa Fails for their support of continuous laryngoscopy during exercise as well as D. Sundstrom and Dusty Christian for their assistance in coordinating the research group.

Author contributions: JT. Olin was responsible for the study inception, study design, data acquisition, and data processing. He wrote the first draft of the manuscript and compiled all author revisions. M. Clary was responsible for study inception, study design, data scoring, and manuscript revision. E. Fan was responsible for data scoring and manuscript revision. K. Johnston was responsible for study inception, study design, data scoring, and manuscript revision. C. State was responsible for data processing, data scoring, and manuscript revision. M. Strand was responsible for all biostatistical calculations and manuscript revision. K. Christopher was responsible for study inception, study design, and manuscript revision.

\section{References}

1 Christensen PM, Heimdal JH, Christopher KL, et al. ERS/ELS/ACCP 2013 international consensus conference nomenclature on inducible laryngeal obstructions. Eur Respir Rev 2015; 24: 445-450.

2 Lakin RC, Metzger WJ, Haughey BH. Upper airway obstruction presenting as exercise-induced asthma. Chest 1984; 86: 499-501.

3 Landwehr LP, Wood RP, Blager FB, et al. Vocal cord dysfunction mimicking exercise-induced bronchospasm in adolescents. Pediatrics 1996; 98: 971-974.

4 McFadden ER Jr, Zawadski DK. Vocal cord dysfunction masquerading as exercise-induced asthma. A physiologic cause for "choking" during athletic activities. Am J Respir Crit Care Med 1996; 153: 942-947.

5 Heimdal JH, Roksund OD, Halvorsen T, et al. Continuous laryngoscopy exercise test: a method for visualizing laryngeal dysfunction during exercise. Laryngoscope 2006; 116: 52-57.

6 Christensen PM, Thomsen SF, Rasmussen N, et al. Exercise-induced laryngeal obstructions: prevalence and symptoms in the general public. Eur Arch Otorhinolaryngol 2011; 268: 1313-1319.

7 Johansson $\mathrm{H}$, Norlander $\mathrm{K}$, Berglund $\mathrm{L}$, et al. Prevalence of exercise-induced bronchoconstriction and exercise-induced laryngeal obstruction in a general adolescent population. Thorax 2015; 70: 57-63. 
Newman KB, Mason UG III, Schmaling KB. Clinical features of vocal cord dysfunction. Am J Respir Crit Care Med 1995; 152: 1382-1386.

9 Komarow HD, Young M, Nelson C, et al. Vocal cord dysfunction as demonstrated by impulse oscillometry. J Allergy Clin Immunol Pract 2013; 1: 387-393.

10 De Guzman V, Ballif CL, Maurer R, et al. Validation of the dyspnea index in adolescents with exercise-induced paradoxical vocal fold motion. JAMA Otolaryngol Head Neck Surg 2014; 140: 823-828.

11 McNally P, Greally P. Use of post-exercise laryngoscopy to evaluate exercise induced dyspnea. Pediatr Pulmonol 2010; 45: 1037-1039.

12 Chiang T, Marcinow AM, deSilva BW, et al. Exercise-induced paradoxical vocal fold motion disorder: diagnosis and management. Laryngoscope 2013; 123: 727-731.

13 Olin JT, Clary MS, Deardorff EH, et al. Inducible laryngeal obstruction during exercise: moving beyond vocal cords with new insights. Phys Sportsmed 2015; 43: 13-21.

14 Tervonen $\mathrm{H}$, Niskanen MM, Sovijarvi AR, et al. Fiberoptic videolaryngoscopy during bicycle ergometry: a diagnostic tool for exercise-induced vocal cord dysfunction. Laryngoscope 2009; 119: 1776-1780.

15 Panchasara B, Nelson C, Niven R, et al. Lesson of the month: Rowing-induced laryngeal obstruction: a novel cause of exertional dyspnoea: characterised by direct laryngoscopy. Thorax 2015; 70: 95-97.

16 Olin JT, Clary MS, Connors D, et al. Glottic configuration in patients with exercise-induced stridor: a new paradigm. Laryngoscope 2014; 124: 2568-2573.

17 Tanner CS, Heise CT, Barber G. Correlation of the physiologic parameters of a continuous ramp versus an incremental James exercise protocol in normal children. Am J Cardiol 1991; 67: 309-312.

18 Dailey SH, Kobler JB, Hillman RE, et al. Endoscopic measurement of vocal fold movement during adduction and abduction. Laryngoscope 2005; 115: 178-183.

19 Roksund OD, Maat RC, Heimdal JH, et al. Exercise induced dyspnea in the young. Larynx as the bottleneck of the airways. Respir Med 2009; 103: 1911-1918.

20 Olin JT, Clary M, Faino A. Clinical significance of observed upper airway obstruction with continuous laryngoscopy during exercise. Am J Respir Crit Care Med 2015; 191: A2121.

21 de Onis M, Onyango AW, Borghi E, et al. Development of a WHO growth reference for school-aged children and adolescents. Bull World Health Organ 2007; 85: 660-667.

22 Ten Harkel A, Takken T. Normal values for cardiopulmonary exercise testing in children. Eur J Cardiovasc Prev Rehabil 2011; 18: 676-677.

23 Baz M, Haji GS, Menzies-Gow A, et al. Dynamic laryngeal narrowing during exercise: a mechanism for generating intrinsic PEEP in COPD? Thorax 2015; 70: 251-257.

24 Maat RC, Roksund OD, Halvorsen T, et al. Audiovisual assessment of exercise-induced laryngeal obstruction: reliability and validity of observations. Eur Arch Otorhinolaryngol 2009; 266: 1929-1936.

25 Christensen P, Thomsen SF, Rasmussen N, et al. Exercise-induced laryngeal obstructions objectively assessed using EILOMEA. Eur Arch Otorhinolaryngol 2010; 267: 401-407.

26 Norlander K, Christensen PM, Maat RC, et al. Comparison between two assessment methods for exercise-induced laryngeal obstructions. Eur Arch Otorhinolaryngol 2016; 273: 425-430.

27 Christensen PM, Maltbaek N, Jorgensen IM, et al. Can flow-volume loops be used to diagnose exercise induced laryngeal obstructions? A comparison study examining the accuracy and inter-rater agreement of flow volume loops as a diagnostic tool. Prim Care Respir J 2013; 22: 306-311.

28 Morris MJ, Christopher KL. The flow-volume loop in inducible laryngeal obstruction: one component of the complete evaluation. Prim Care Respir J 2013; 22: 267-268. 